\title{
Effect of Yin-Xing-Tong-Zhi Tablets on Improving Vascular Cognitive Impairment No Dementia
}

\author{
Weidong Pan $\left(\mathbb{D},{ }^{1}\right.$ Wei Zhu, ${ }^{2}$ Qi Gao, ${ }^{3}$ Weilong Liao, ${ }^{1}$ Penglin Gao, ${ }^{1}$ and Te Liu ${ }^{4}{ }^{4}$ \\ ${ }^{1}$ Department of Neurology, Shuguang Hospital Affiliated to Shanghai University of Traditional Chinese Medicine, Shanghai, China \\ ${ }^{2}$ Department of Neurology, Gongli Hospital, The Second Military Medical University, Shanghai, China \\ ${ }^{3}$ SPH Xingling Technology Pharmaceutical Co., Ltd., Shanghai, China \\ ${ }^{4}$ Shanghai Geriatric Institute of Chinese Medicine, Longhua Hospital, Shanghai University of Traditional Chinese Medicine, \\ Shanghai, China
}

Correspondence should be addressed to Weidong Pan; panwd@medmail.com.cn and Te Liu; liute1979@126.com

Received 30 December 2017; Accepted 26 March 2018; Published 26 April 2018

Academic Editor: José L. Rios

Copyright (C) 2018 Weidong Pan et al. This is an open access article distributed under the Creative Commons Attribution License, which permits unrestricted use, distribution, and reproduction in any medium, provided the original work is properly cited.

Objective. Observe the effect of the Chinese herbal extracts, Yin-Xing-Tong-Zhi, in tablet form, on improving vascular cognitive impairment no dementia (VCIND). Methods. Sixty-eight patients with VCIND were divided randomly into treatment and placebo groups with oral administration of Yin-Xing-Tong-Zhi tablets (YXTZTs) or placebo, respectively, for 24 weeks. Alzheimer's Disease Assessment Scale-Cognitive (ADAS-Cog) subscale score, MMSE score, Clinician's Interview-Based Impression of Change Plus Caregiver Input (CIBIC-Plus) score, expression of interleukin- (IL-) 6, IL-8, and tumor necrosis factor- (TNF-) $\alpha$ in serum, and variation of blood-lipid levels were evaluated at different time points. Results. At weeks 12 and 24, the scores for the ADAS-Cog, CIBIC-Plus, and MMSE of the treatment group were significantly lower than those of the control group $(P<0.05)$. All clinical scales at week 24 of the control group were significantly different from those before treatment $(P<0.05)$. Expression of IL-6, IL-8, and TNF- $\alpha$ in the two groups was reduced significantly with variation of the clinical scales of cognitive impairment. Conclusion. YXTZTs may delay the development of cognitive impairment in VCIND patients by modulating expression of VCIND-associated proinflammatory factors.

\section{Introduction}

Vascular cognitive impairment without dementia (VCIND) is an early stage of vascular dementia $(\mathrm{VaD})[1,2]$. The main symptoms can be a decline in understanding, execution, memory, visual spatial skills, and emotions of varying severities and can involve damage to the posterior cingulate cortex and frontal lobe of the brain [3].

It has been accepted that effective treatment could delay the progression of VCIND to VaD [4]. Early studies indicated using special traditional Chinese medicine (TCM) formulas may delay the development or improve the pathologic changes of $\mathrm{VaD}$ or $\mathrm{AD}[5,6]$. Hence, the early diagnosis and treatment of VCIND is very important [7]. Pharmacologic treatment of VCIND is lacking, as are efficacy studies using TCM for VCIND.

Yin-Xing-Tong-Zhi tablets (YXTZTs) are made from the extracts of Yin-Xing-Tong-Zhi, the active ingredients of which are flavone glycosides and terpene lactones [8]. Xiaojian and colleagues showed that YXTZTs can reduce the prevalence of vascular cognitive impairment [9]. However, no scholars have used YXTZTs to prevent or treat cognitive impairment. According to the mechanism of action of YXTZTs in cerebrovascular diseases (CVDs) [10], it can delay VCIND progression and prevent VaD. Here, we conducted a clinical study using YXTZTs in the treatment of VCIND.

\section{Materials and Methods}

2.1. Ethical Approval of the Study Protocol. The protocol for our randomized double-blind study was approved by the Ethics Committee of Shuguang Hospital (affiliated to the Shanghai University of Traditional Chinese Medicine, Shanghai, China) and was in accordance with the Declaration of the Helsinki. All patients provided written informed consent to participate in our study. 
TABLE 1: Baseline characteristics of the two groups before treatment.

\begin{tabular}{|c|c|c|c|c|}
\hline & YXTZTs $(n=34)$ & Placebo $(n=34)$ & $X 2$ or $t$ or $Z$ & $P$ \\
\hline Male/female & $20 / 14$ & $26 / 8$ & 2.419 & 0.120 \\
\hline Age & $61.76 \pm 5.33$ & $62.69 \pm 4.32$ & 0.450 & 0.654 \\
\hline Education $(\mathrm{M} / \mathrm{H} / \mathrm{C})$ & $3 / 28 / 3$ & $4 / 29 / 1$ & 3.212 & 0.201 \\
\hline Baseline of ADAS-COG & $12.74 \pm 2.48$ & $12.76 \pm 2.71$ & 0.540 & 0.589 \\
\hline Baseline of CIBIC-PLUS & $13.09 \pm 2.56$ & $13.15 \pm 1.65$ & 1.778 & 0.075 \\
\hline Baseline of MMSE & $24.00(23.00,25.00)$ & $24.00(24.00,25.00)$ & -0.409 & 0.682 \\
\hline Baseline of IL- 6 & $4.81(2.54,14.60)$ & $6.00(4.09,12.00)$ & -0.933 & 0.351 \\
\hline Baseline of IL-8 & $25.70(11.28,39.13)$ & $18.00(12.23,35.20)$ & -0.276 & 0.783 \\
\hline Baseline of TNF- $\alpha$ & $12.70(8.28,17.98)$ & $14.00(12.00,15.23)$ & -1.086 & 1.277 \\
\hline
\end{tabular}

Note. YXTZTs: Yin-Xing-Tong-Zhi tablets; M: middle school; H: high school; C: college; ADAS-Cog: Alzheimer's Disease Assessment Scale-Cognitive subscale; CIBIC-Plus: Clinician Interview based Impression of Severity; MMSE: Mini-Mental State Examination; IL-6: interleukin-6; IL-8: interleukin-8; TNF- $\alpha$ : tumor necrosis factor.

2.2. Diagnostic Criteria. We combined the diagnostic criteria for VCIND formulated by Ingles et al. [4] with the recommendations of Jianping and colleagues for vascular cognitive impairment [11]. The criteria for VCIND were as follows: CVDs or CVD risk factors; fluctuating cognitive impairment; no/mild memory loss; no other severe diseases; causal relationship between CVD and cognitive impairment; ability to carry out the activities of daily living; not meeting the diagnostic criteria for dementia.

2.3. Inclusion and Exclusion Criteria. Inclusion criteria were as follows: (i) meeting the diagnostic criteria stated above; (ii) age, 50-70 years; (iii) use of at least one rating scale for clinical dementia, for example, a Mini-Mental State Examination (MMSE) score $\geq 20$ for individuals who completed primary education or $\geq 24$ for those who completed secondary or higher education; (iv) magnetic resonance imaging suggesting subcortical ischemic CVD in the brain.

Exclusion criteria were as follows: (i) patients with other diseases that could cause dementia (e.g., multiple sclerosis, cerebral hemorrhage, watershed infarction, atrophy of the hippocampus or olfactory nucleus); (ii) a Hamilton Depression Scale score > 17; (iii) severe neurologic deficits (e.g., aphasia, malaise, and severe hemiplegia); (iv) severe primary diseases of the heart, kidney, endocrine, or hematopoietic systems; (v) mental illness or severe epilepsy; (vi) allergies or allergy to the study drug.

2.4. Patient Characteristics. Sixty-eight patients with VCIWD who were inpatients or outpatients from the Department of Neurology of Shuguang Hospital from October 2015 to March 2017 formed the study cohort. Observations ended in August 2017. There were 46 males and 22 females (35-76 years (mean, $52.8 \pm 5.9)$ ). The general characteristics of the patients, their clinical-rating scales before study inclusion, and laboratory-related tests are shown in Table 1 . All the patients were diagnosed by very experienced neurologists.

2.5. Patient Randomization. SPSS v20.0 (IBM, Armonk, NY, USA) was used to generate random-number tables and group numbers at a ratio of $1: 1$ in accordance with the method of complete randomization. Shanghai Xingling Technology
Pharmaceuticals were commissioned to produce YXTZTs and placebo according to group numbers. Participants were given the corresponding test drugs according to the order they were included in the clinical observation. The clinical observers and patients did not know the grouping of drugs. Two groups of patients (VCIWD and placebo) were created.

2.6. Therapeutic Method. To ensure that patients could manage their comorbidities, they were given the relevant basic treatment. Patients with cerebral infarction were given antiplatelet agents. Cases with hypertension were given calcium antagonists, $\beta$-blockers, angiotensin-converting enzyme inhibitors, or angiotensin II receptor-blockers. Patients suffering from diabetes mellitus were given insulin secretagogues, biguanides, $\alpha$-glucosidase inhibitors, insulin sensitizers, or insulin. Cases suffering from hyperlipidemia were given statins or fibrates.

Besides the basic treatment stated above, the treatment group were given (at the same time) one YXTZT (t.d.s.). Besides their basic treatment, the control group were given a placebo tablet of the same shape as a YXTZT (t.d.s.). The treatment lasted for 24 weeks. Patients came to a neurology clinic every 2 weeks for further consultation and to obtain YXTZTs/placebos.

2.7. Outcome Measures. The primary outcome measures were the Alzheimer's Disease Assessment Scale-Cognitive subscale (ADAS-Cog) score, MMSE score, and the Clinician's Interview-Based Impression of Change Plus Caregiver Input (CIBIC-Plus) score at the day of study inclusion, week 12 , and week 24.

The secondary outcome measures were serum levels of interleukin- (IL-) 6, IL-8, and tumor necrosis factor- (TNF-) $\alpha$ at the day of study inclusion, week 12, and week 24 .

We also measured blood levels of lipids (total cholesterol, high-density lipoprotein, low-density lipoprotein, and triglycerides) and undertook blood biochemical analyses. The urine/stool routine was documented, liver and kidney function noted, and electrocardiography undertaken. These parameters were tested before and after treatment. Also, adverse drug events were monitored. 


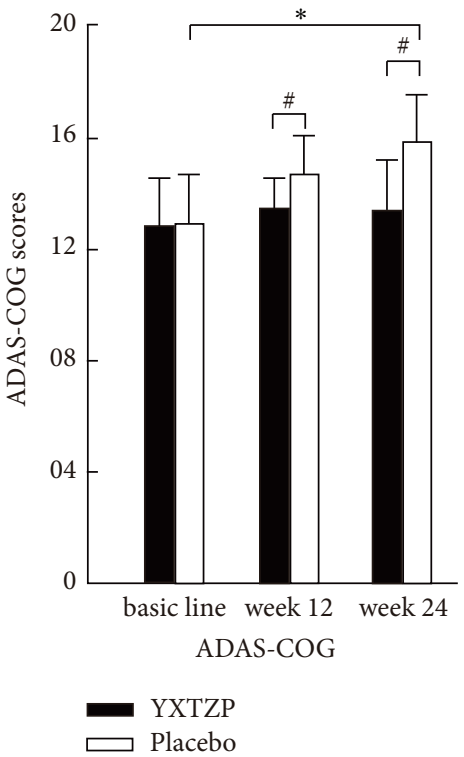

(a)

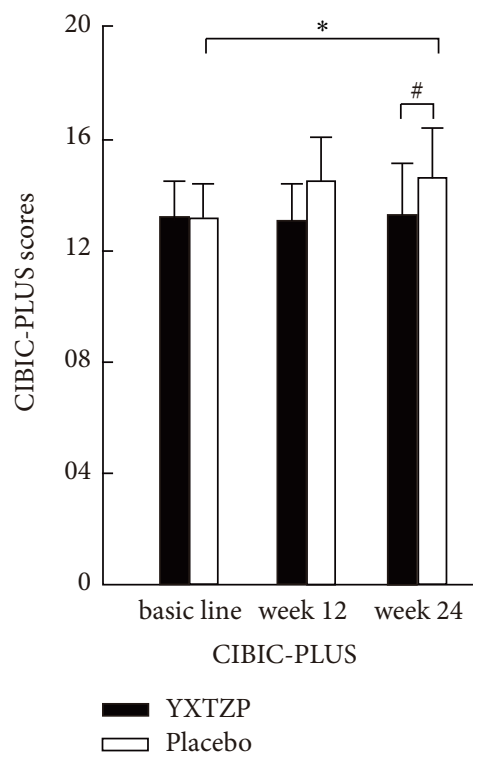

(b)

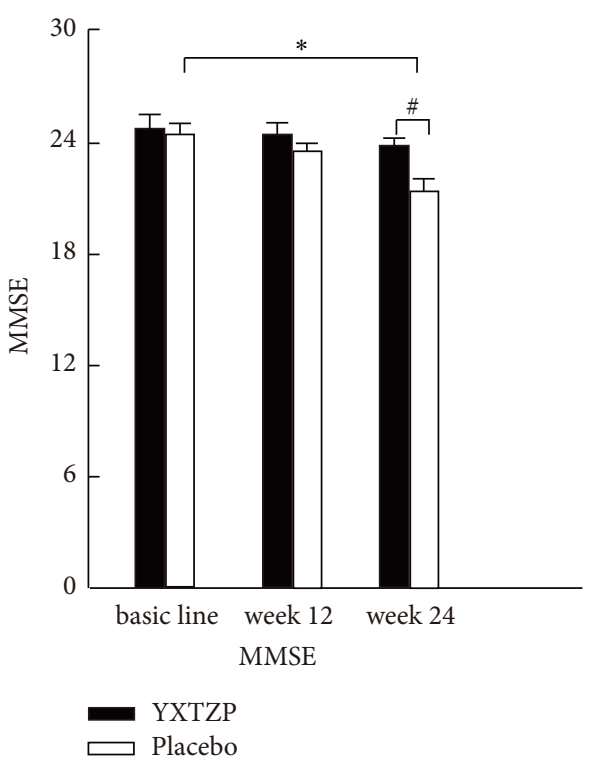

(c)

FIGURE 1: Comparison of clinical scales of the two groups before and after treatment. ${ }^{\#} P<0.05$, comparison between the two groups; ${ }^{*} P<0.05$, comparison of the same group before and after treatment; YXTZP: Yin-Xing-Tong-Zhi tablets; ADAS-Cog: Alzheimer's Disease Assessment Scale-Cognitive subscale; CIBIC-Plus: Clinician Interview-based Impression of Severity; MMSE: Mini-Mental State Examination.

2.8. Statistical Analyses. Data are the mean \pm SD. All statistical tests were two-sided. Changes in parameters in the two groups before and after treatment were compared using ANOVA and the Wilcoxon rank sum test, as well as the $\chi^{2}$ test or nonparametric test. $P<0.05$ was considered significant. SPSS v20.0 was employed for statistical analyses.

\section{Results}

3.1. Demographics. Sixty-eight patients with VCIWD formed the study cohort. Thirty-four patients were in the treatment group and 34 in the placebo group. All 68 patients completed the study.

There were no significant differences in sex, marital status, age, educational level, blood lipids, clinical scale score before treatment, or expression of proinflammatory factors between the two groups (Table 1). Significant liver or kidney dysfunction or significant drug-related adverse events were not observed in either group during treatment.

3.2. Primary Outcome Measures. Compared with the ADASCog scores before treatment, the ADAS-Cog scores at week 12 and week 24 in the treatment group increased, but the differences were not significant $(P>0.05)$. Compared with the ADAS-Cog scores before treatment, the ADASCog scores of the control group increased at week 12 week $(P>0.05)$, and the differences were not significant, but the increased scores at week 24 were not significantly different from those before treatment $(P<0.05)$. At week 24 , the ADAS-Cog scores were significantly different between the two groups $(P<0.05)$ (Table 1 , Figure $1(\mathrm{a}))$.

Compared with the CIBIC-Plus scores before treatment, the CIBIC-Plus scores at week 12 and week 24 in the treatment group decreased, but the differences were not significant $(P>$ 0.05). However, the CIBIC-Plus scores at week 12 and week 24 in the control group increased, and the differences were not significant compared with those before treatment $(P>0.05)$. The difference value was significantly different between the two groups at week 12 and week $24(P<0.05)$ (Figure $1(\mathrm{~b})$ ).

Compared with the MMSE scores before treatment, the MMSE scores at week 12 and week 24 in the treatment group decreased, but the differences were not significant $(P>0.05)$. The MMSE score at week 12 in the control group decreased, and the difference was not significantly different compared with that before treatment $(P>0.05)$. Also, the MMSE score at week 24 decreased, and the difference was significantly different compared with that before treatment $(P<0.05)$. The $d$ value was significantly different between the two groups at week $24(P<0.05)$ (Figure 1$)$.

The data shown above suggested that the cognitive function of the two groups decreased but, at week 24, the cognitive function of the control group decreased more severely.

3.3. Secondary Outcome Measures. In the treatment group, IL-6 expression decreased at week 12 compared with that before treatment, but the difference was not significant $(P>0.05)$. IL-6 expression decreased at week 24 , but the difference was significant $(P<0.05)$. In the control group, IL-6 expression decreased at 12 weeks and 24 weeks after treatment, but the difference was not significant $(P>0.05)$. At week 24, the $d$ value was significantly different between the two groups $(P<0.05)$ (Figure $2(\mathrm{a}))$.

In the treatment group, IL-8 expression decreased at week 12 compared with that before treatment, but the difference was not significant $(P>0.05)$. IL-8 expression decreased at week 24 , and the difference was significant $(P<0.05)$. In 


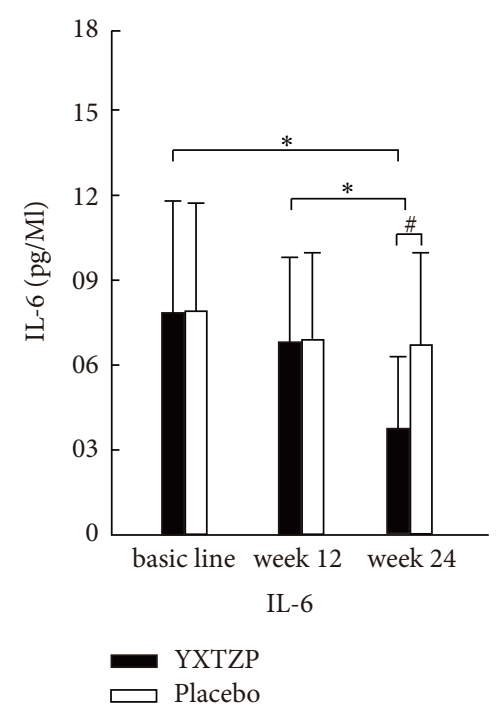

(a)

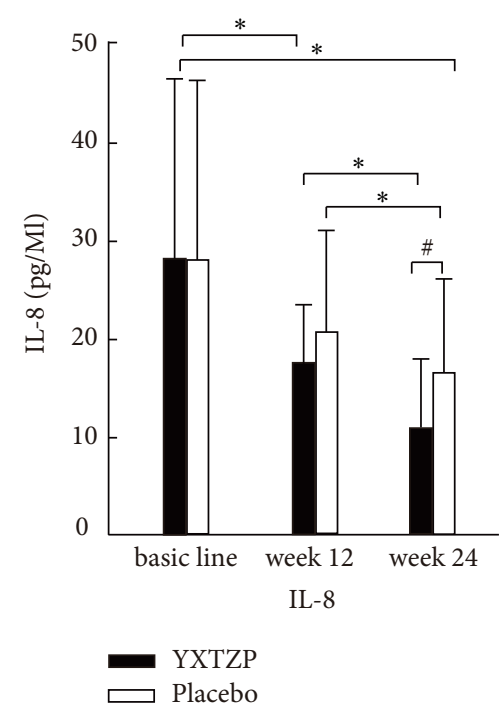

(b)

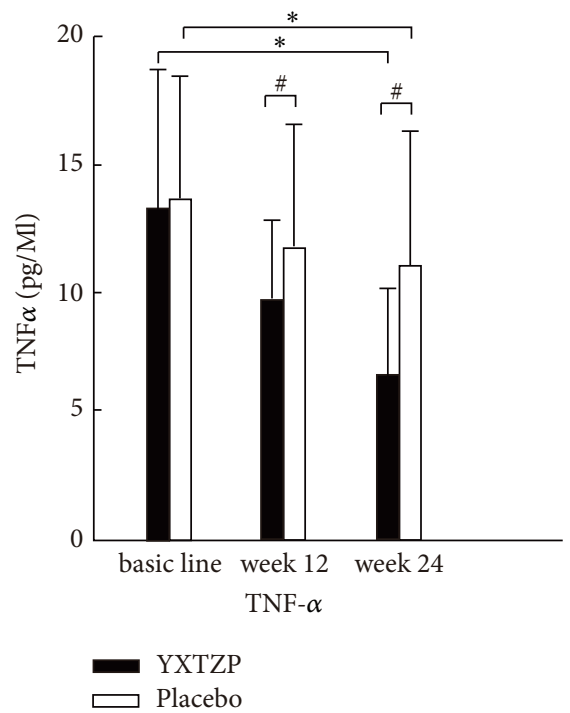

(c)

Figure 2: Comparison of changes in levels of proinflammatory factors between the two groups before and after treatment. ${ }^{\#} P<0.05$, comparison between the two groups; ${ }^{*} P<0.05$, comparison of the same group before and after treatment. YXTZP: Yin-Xing-Tong-Zhi tablets; IL-6: interleukin-6; IL-8: interleukin-8; TNF- $\alpha$ : tumor necrosis factor.

the control group, IL-8 expression increased 12 weeks after treatment, but the difference was not significant $(P>0.05)$. IL-8 expression decreased 24 weeks after treatment, and the difference was significant $(P<0.05)$ (Figure 2(b)).

Compared with TNF- $\alpha$ expression before treatment, TNF- $\alpha$ expression at week 12 and week 24 in the treatment group decreased significantly, and the difference was significant $(P<0.05)$. In the control group, TNF- $\alpha$ expression at week 12 decreased, but the difference was not significant $(P>0.05)$. TNF- $\alpha$ expression at week 24 decreased, and the difference was significant $(P<0.05)$ (Figure $2(\mathrm{c}))$. The $d$ value of TNF- $\alpha$ between the two groups was significant at week 12 and week $24(P<0.05)$.

\section{Discussion}

VCIND is the early stage of VaD and is caused primarily by cerebral arteriosclerosis and ischemic stroke. Hyperlipidemia and the viscosity of cerebral blood flow are important pathogenic factors of cerebral atherosclerosis. VCIND is similar to the pathogenesis of vascular cognitive impairment or $\mathrm{VaD}[12]$.

From a TCM perspective, VCIND can be attributed to categories such as "dementia" or "amnesia." It is a mental disorder with abnormal symptoms caused by physiologic dysfunction, deficiency of essence qi, brain dystrophy, stasis, and other pathogenic factors. The main clinical manifestations are low intelligence and amnesia. Less severe VCIND can result in patients appearing indifferent, less talkative, unresponsive, and forgetful. Combining ancient with modern medical knowledge, we hypothesized that VCIND is an early stage of dementia and, at this stage, drug treatment could help to delay its development.

We conducted a study to ascertain the clinical efficacy of YXTZTs on VCIND. We found that the rate of progression of clinical scales that reflect cognitive function in VCIND patients was delayed significantly after taking YXTZTs for 24 weeks compared with placebos (Figure 1). These differences in efficacy may have involved changes in expression of some proinflammatory factors in atherosclerotic and vascular lesions (Figure 2). Changes in these clinical scales were not related directly to the lipid profiles of VCIND patients.

YXTZTs are used widely in TCM. The preparations within YXTZTs are important for the treatment of CVDs and cardiovascular diseases. These preparations are also used as vegetable supplements in several European countries [13]. TCM theory holds that YXTZ extracts can promote blood circulation to remove blood stasis and can dredge meridians and collaterals. The active ingredients in YXTZTs are flavone glycosides and terpene lactones [14], which can activate the blood circulation to remove blood stasis and open orifices, which in TCM is termed "blood-activating and "orificeopening."

Several clinical and basic research studies have shown that YXTZ extracts can improve the symptoms and delay the progression of dementia, improve intelligence levels, and aid the activities of daily living [15-19]. Basic research studies [20] have also suggested that YXTZ extracts can improve the learning and memory of mice with senile dementia and increase the number of hippocampal pyramidal cells in their brain. Studies on $\mathrm{VaD}$ have shown good curative effects using YXTZ extracts, and some scholars have speculated that these effects are related to the functions of proinflammatory factors and choline lipase in the brain [21-23]. Hence, YXTZ extracts could aid in helping to delay vascular cognitive impairment.

Studies have shown that expression of proinflammatory markers in the peripheral circulation increases before dementia onset [23]. IL-6 is an important multiple-effect cytokine that has an important role in the immune response. The way that IL-6 affects learning and memory may involve 
synaptic plasticity and neurogenesis [24]. IL-8 promotes angiogenesis, which has a positive correlation with dementia severity. The mechanism of $\mathrm{VaD}$ correlates with increases in the numbers of immune cells and the inflammatory response [25]. TNF- $\alpha$ can inhibit neuroinflammatory reactions in rats with $\mathrm{VaD}$ and enhance vascular repair to protect the brain [26]. Expression of these proinflammatory cytokines can, to a certain extent, reflect the extent to which the brain suffers cognitive impairment due to vascular factors. Expression of IL-6, IL-8, and TNF- $\alpha$ in the present study decreased over time, but it decreased more significantly compared with control patients who took YXTZTs. Cognitive-related symptoms were delayed, but we did not undertake correlation analyses, which was a shortcoming of our study. Another limitation was the small study cohort.

One strength of the present study was that it focused on the early stage of vascular cognitive impairment: VaD. The latter is a nondementia stage during which effective intervention could delay the progression of cognitive impairment. Current thinking is that, irrespective of the type of dementia, once it progresses, it becomes irreversible and will continue to worsen slowly $[7,11]$. Our study showed that effective intervention at the early stage of dementia, and treatment for 24 weeks, delayed VCIND progression. Our study showed the slow, stable, and reliable effects of Chinese medicines in the treatment of diseases.

\section{Conclusions}

This clinical study demonstrated that VCIND can be treated and relieved by activating blood and opening orifices. YXTZTs can delay the development of cognitive impairment in VCIWD patients and may alleviate the worsening of VCIND by regulating the associated proinflammatory factors, but there was no significant correlation with blood-lipid profiles.

\section{Disclosure}

Weidong Pan and Wei Zhu are co-first authors.

\section{Conflicts of Interest}

The authors declare that there are no conflicts of interest regarding the publication of this article.

\section{Acknowledgments}

The present study was supported by a grant from The National Natural Science Foundation of China (Grants nos. 81373619 and 81202811).

\section{References}

[1] Expert consensus on vascular cognitive impairment, "Expert understanding of vascular cognitive impairment," Chinese Journal of Internal Medicine, vol. 46, no. 12, pp. 1052-1054, 2007.

[2] P. B. Gorelick and L. Pantoni, "Advances in vascular cognitive impairment," Stroke, vol. 44, no. 2, pp. 307-308, 2013.
[3] S. Black and C. Iadecola, "Vascular cognitive impairment: Small vessels, big toll introduction," Stroke, vol. 40, no. 3, pp. S38-S39, 2009.

[4] J. L. Ingles, C. Wentzel, J. D. Fisk, and K. Rockwood, "Neuropsychological predictors of incident dementia in patients with vascular cognitive impairment, without dementia," Stroke, vol. 33, no. 8, pp. 1999-2002, 2002.

[5] J. Jia, C. Wei, J. Liang et al., "The effects of DL-3-n-butylphthalide in patients with vascular cognitive impairment without dementia caused by subcortical ischemic small vessel disease: A multicentre, randomized, double-blind, placebo-controlled trial," Alzheimer's \& Dementia, vol. 12, no. 2, pp. 89-99, 2016.

[6] G.-R. Zhang, X.-R. Cheng, W.-X. Zhou, and Y.-X. Zhang, "Agerelated expression of calcium/calmodulin-dependent protein kinase II A in the hippocampus and cerebral cortex of senescence accelerated mouse prone/ 8 mice is modulated by antiAlzheimer's disease drugs," Neuroscience, vol. 159, no. 1, pp. 308315, 2009.

[7] X.-R. Cheng, X.-L. Cui, Y. Zheng et al., "A co-module regulated by therapeutic drugs in a molecular subnetwork of alzheimer's disease identified on the basis of traditional chinese medicine and samp8 mice," Current Alzheimer Research, vol. 12, no. 9, pp. 870-885, 2015.

[8] X. J. Xiong, W. Liu, X. C. Yang et al., "Ginkgo biloba extract for essential hypertension: a systemic review," Phytomedicine, vol. 21, no. 10, pp. 1131-1136, 2014.

[9] W.-Z. Li, W.-Y. Wu, H. Huang, Y.-Y. Wu, and Y.-Y. Yin, "Protective effect of bilobalide on learning and memory impairment in rats with vascular dementia," Molecular Medicine Reports, vol. 8, no. 3, pp. 935-941, 2013.

[10] H. Satoh, "Comparative Electropharmacological Actions of Some Constituents from Ginkgo biloba Extract in Guinea-pig Ventricular Cardiomyocytes," Evidence-Based Complementary and Alternative Medicine, vol. 1, no. 3, pp. 277-284, 2004.

[11] A. Consoli, M. Pasi, and L. Pantoni, "Vascular mild cognitive impairment: concept, definition, and directions for future studies," AGING, vol. 24, no. 2, pp. 113-116, 2012.

[12] G. Teichner and M. T. Wagner, "The Test of Memory Malingering (TOMM): Normative data from cognitively intact, cognitively impaired, and elderly patients with dementia," Archives of Clinical Neuropsychology, vol. 19, no. 3, pp. 455-464, 2004.

[13] B. Zheng, L. Teng, G. Xing et al., "Proliposomes containing a bile salt for oral delivery of Ginkgo biloba extract: Formulation optimization, characterization, oral bioavailability and tissue distribution in rats," European Journal of Pharmaceutical Sciences, vol. 77, article no. 3291, pp. 254-264, 2015.

[14] W. Dandan, Y. Yujiao, J. Wang, W. Yafang et al., "Development of fingerprint atlas analysis method of flavonoids in Ginkgo Biloba," Chinese Journal of New Drugs, vol. 10, pp. 1194-1199, 2017.

[15] J. Kobus-Cisowska, E. Flaczyk, M. Rudzińska, and D. Kmiecik, "Antioxidant properties of extracts from Ginkgo biloba leaves in meatballs," Meat Science, vol. 97, no. 2, pp. 174-180, 2014.

[16] N. T. Lautenschlager, R. Ihl, and W. E. Müller, “Ginkgo biloba extract EGb 761 in the context of current developments in the diagnosis and treatment of age-related cognitive decline and Alzheimer's disease: a research perspective," International Psychogeriatrics, vol. 24, supplement 1, pp. S46-S50, 2012.

[17] M.-N. Rocher, D. Carré, B. Spinnewyn et al., "Long-term treatment with standardized Ginkgo biloba Extract (EGb 761) attenuates cognitive deficits and hippocampal neuron loss in a 
gerbil model of vascular dementia," Fitoterapia, vol. 82, no. 7, pp. 1075-1080, 2011.

[18] T. Sun, X. Wang, and H. Xu, " Ginkgo Biloba extract for angina pectoris: A systematic review," Chinese Journal of Integrative Medicine, vol. 21, no. 7, pp. 542-550, 2015.

[19] P. Montes, E. Ruiz-Sánchez, C. Rojas, and P. Rojas, "Ginkgo biloba extract 761: A review of basic studies and potential clinical use in psychiatric disorders," CNS and Neurological Disorders - Drug Targets, vol. 14, no. 1, pp. 132-149, 2015.

[20] N. A. Boghdady, "Antioxidant and antiapoptotic effects of proanthocyanidin and Ginkgo biloba extract against doxorubicin-induced cardiac injury in rats," Cell Biochemistry \& Function, vol. 31, no. 4, pp. 344-351, 2013.

[21] H.-F. Zhang, L.-B. Huang, Y.-B. Zhong et al., "An overview of systematic reviews of Ginkgo biloba extracts for mild cognitive impairment and dementia," Frontiers in Aging Neuroscience, vol. 8, article no. 276, 2016.

[22] R. B. Silberstein, A. Pipingas, J. Song, D. A. Camfield, P. J. Nathan, and C. Stough, "Examining brain-cognition effects of ginkgo biloba extract: brain activation in the left temporal and left prefrontal cortex in an object working memory task," Evidence-Based Complementary and Alternative Medicine, vol. 2011, Article ID 164139, 10 pages, 2011.

[23] J.-S. Chen, Y.-H. Chen, P.-H. Huang et al., "Ginkgo biloba extract reduces high-glucose-induced endothelial adhesion by inhibiting the redox-dependent interleukin-6 pathways," Cardiovascular Diabetology, vol. 11, article no. 49, 2012.

[24] C.-H. Lu, C.-W. Hwang, N.-F. Chen, W.-S. Liu, Y.-F. Hsiao, and W.-T. Wu, "In vivo effects of Ginkgo biloba extract on interleukin-6 cytokine levels in patients with neurological disorders," Indian Journal of Pharmacology, vol. 44, no. 1, pp. 118-121, 2012.

[25] S. Trompezinski, M. Bonneville, I. Pernet, A. Denis, D. Schmitt, and J. Viac, "Gingko biloba extract reduces VEGF and CXCL8/IL-8 levels in keratinocytes with cumulative effect with epigallocatechin-3-gallate," Archives of Dermatological Research, vol. 302, no. 3, pp. 183-189, 2010.

[26] Y. B. Jiao, Y. C. Rui, P. Y. Yang, T. J. Li et al., "Effects of Ginkgo biloba extract on expressions of IL-1beta, TNF-alpha, and IL-10 in U937 foam cells," Yao Xue Xue Bao, vol. 42, no. 9, pp. 930-934, 2007. 


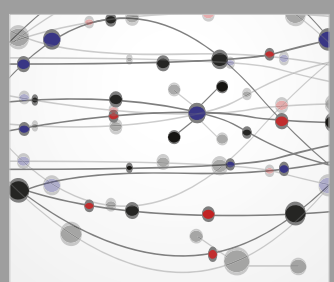

The Scientific World Journal
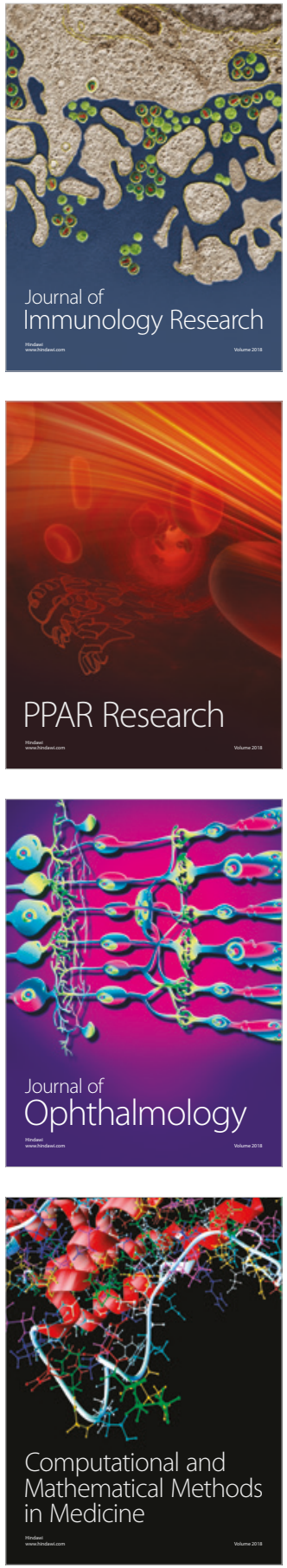

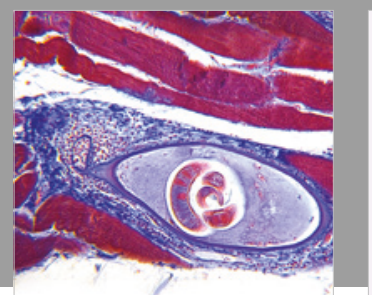

Gastroenterology Research and Practice

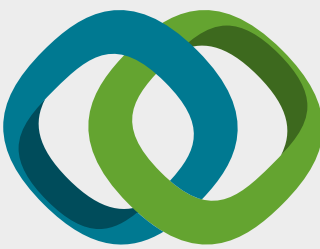

\section{Hindawi}

Submit your manuscripts at

www.hindawi.com
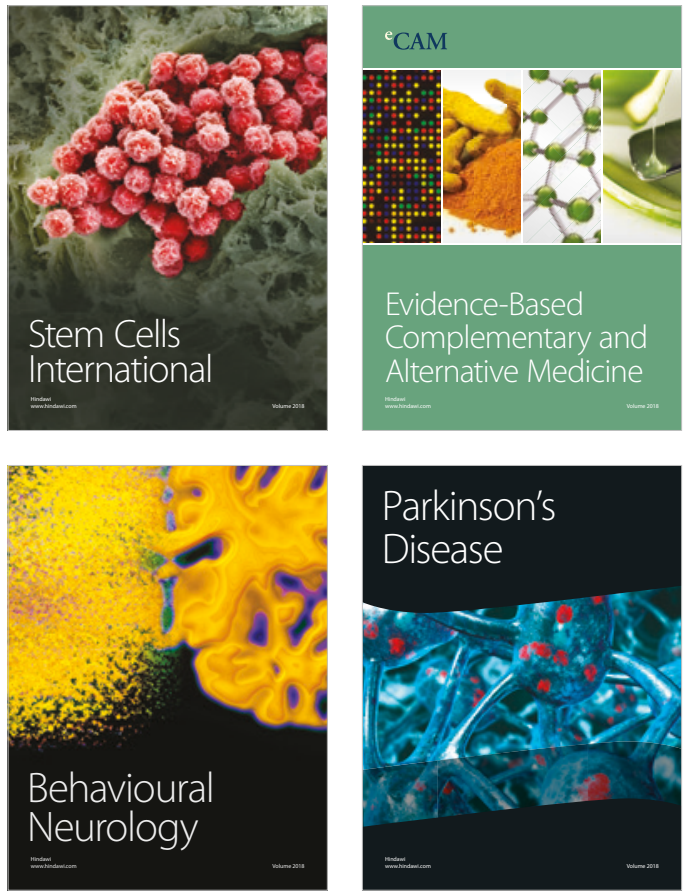

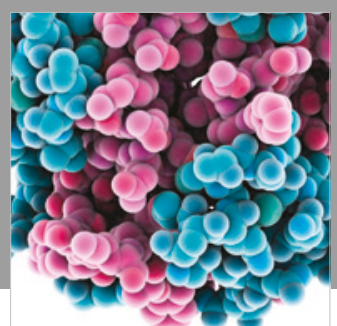

ournal of

Diabetes Research

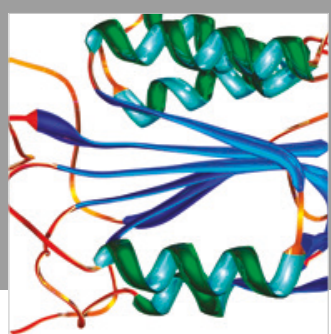

Disease Markers
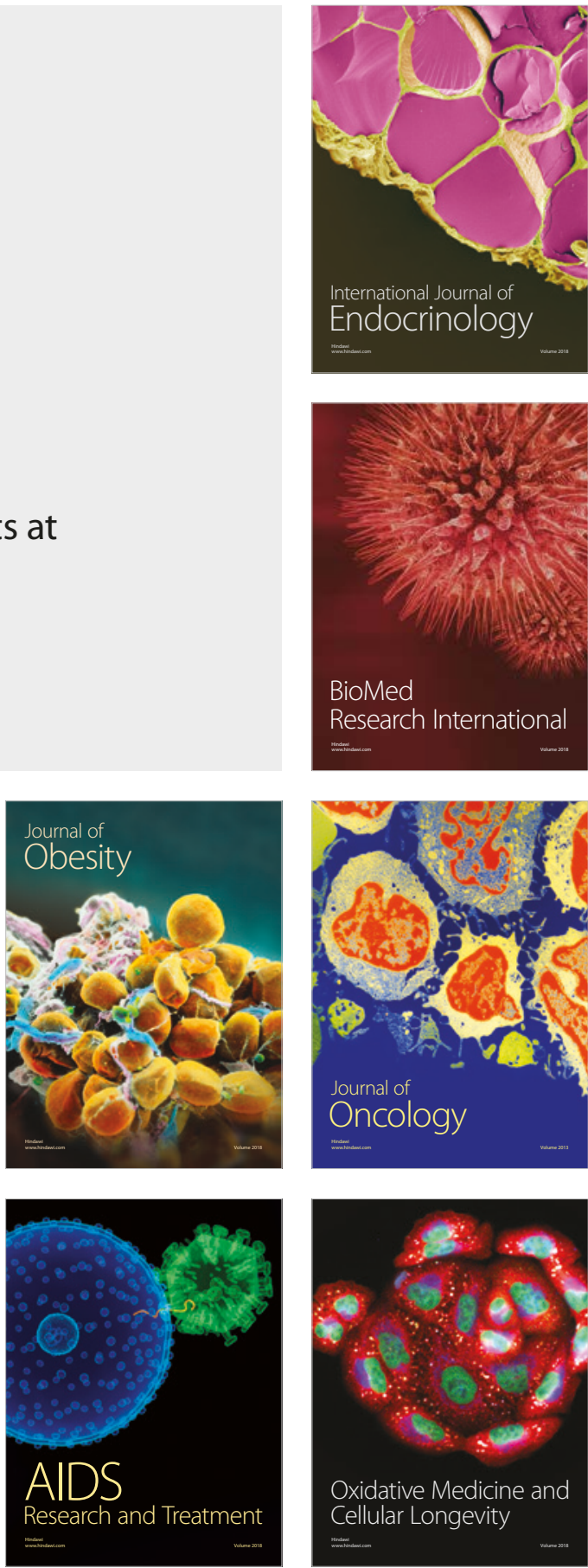